\title{
Provision of emergency contraception in general practice and confidentiality for the under 16's: Results of a postal survey by general practitioners in Avon
}

\author{
Anna Graham, MSc, MRCGP, DFFP, Clinical Research Fellow, Division of Primary Health Care, University of Bristol, Bristol, \\ UK; Laurence Moore, MSc, PhD, Senior Research Fellow, Cardiff University School of Social Sciences, Cardiff, UK; Deborah \\ Sharp, PhD, FRCGP, Professor of Primary Health Care, Division of Primary Health Care, University of Bristol, Bristol, UK.
}

Correspondence: Dr A Graham, Clinical Research Fellow, Division of Primary Health Care, University of Bristol, Canynge Hall, Whiteladies Road, Bristol BS8 2PR. Tel: 0117928 7205. Email: a.graham@ bristol.ac.uk

(Accepted $8^{\text {th }}$ March 2001)

The Journal of Family Planning and Reproductive Health Care 2001: 27(4): 193-196

\begin{abstract}
Objective. To describe the provision of emergency contraception and confidentiality for the under 16's by general practitioners (GPS) in Avon, in order to inform the development of a health promotion intervention in schools in Avon.
\end{abstract}

Design. Confidential postal questionnaire survey.

Setting. All principals in general practice in Avon Health Authority, South West England.

Subjects. Five hundred and eighty general practice principals were sent the questionnaire.

Results. Four hundred and eighty-six (84\%) principals in general practice responded to the questionnaire. Only three (0.6\%) GPs did not provide hormonal emergency contraception. Nearly half $(232,47.7 \%)$ would fit the intrauterine device (IUD) as emergency contraception. Fitting an IUD was associated with female gender of the GP (OR $=2.34,95 \%$ CI 1.53-3.71), and whether the GP had a family planning qualification $(O R=4.55$, 95\% CI 2.418.60). Three hundred and fifty-two (72\%) respondents would provide emergency contraception on a Sunday if requested to do so by a 14-year-old who reported having had unprotected sex the night before.

Practice nurses in 26 (5\%) of the respondent's practices were available to provide advice and tablets for patients requesting hormonal emergency contraception. However, $74(21 \%)$ respondents employed a family planning trained practice nurse who was not involved in any way in the provision of emergency contraception. Practice nurses remain an under used resource in this area.

Conclusion. Our findings suggest that most GPs provide hormonal emergency contraception. Only eight (1.6\%) of respondents would need to ask for parental consent prior to providing hormonal emergency contraception to a 14-year old-girl. Young people need to be informed of GPS widespread adherence to current confidentiality guidelines.

\section{Key message points}

- Most general practitioners (GPs) in Avon provide hormonal emergency contraception (EC) and nearly half say they would fit a coil as EC

- Two thirds of GPs in this sample provide EC within 24 hours of unprotected sex, if asked on a Sunday.

- Practice nurses are an under used resource in the provision of EC.

- Almost all GPs in this study would maintain confidentiality if a 14-year-old asked them for EC.

- Practices need to publicise their provision of a confidential contraceptive advice service.

\section{Introduction}

In the period 1995-1997 in England and Wales there were 45 conceptions per thousand women aged 15-17 years, the highest in Western Europe. ${ }^{1}$ Halving the rate of conceptions among under 18-year-olds in England by 2010 is a government priority. ${ }^{2}$ Age at first intercourse has fallen in Britain over the past four decades, ${ }^{3}$ and research has shown that if young people have sexual intercourse before the age of 16 they are less likely to use contraception than if first intercourse occurs at a later age. ${ }^{4}$ Condoms are increasingly being used as a sole method of contraception.

Emergency contraception (EC) is a safe,${ }^{5}$ effective,${ }^{6}$ and economic ${ }^{7}$ method of preventing pregnancy. EC can be used if there is a condom accident, or if no method of contraception is used during intercourse. Hormonal EC is more effective if given within 24 hours of unprotected sexual intercourse. ${ }^{6}$ Access to EC is a problem for young people and is limited to general practice in some parts of the country, and in others general practice is the only provider at weekends. The main barrier to use of general practice by young people for sexual health problems is confidentiality. ${ }^{8}$

A postal survey was devised aiming to describe the provision of EC by GPs in the area in order to inform the development of a health promotion intervention in schools in Avon. We report the results of this survey.

\section{Method}

A questionnaire was developed to provide information on the provision of EC in general practice, and to explore GPs' attitudes towards confidentiality in the under 16's. A review of the literature ${ }^{8-10}$ and discussion with colleagues in general practice and family planning indicated the most important areas to cover. The questionnaire was piloted on a random sample of 24 GPs from six practices in Oxfordshire. The pilot assessed how long the questionnaire took to complete, clarity of the questions and the GPs' views on all partners in the same practice receiving the same questionnaire. The process was acceptable to the pilot GPs with minor changes made to the questionnaire.

A final version of the confidential questionnaire was sent to all 580 GP principals on the Avon Health Authority's database in May 1999. Non-responders were sent a second questionnaire 2 weeks after the first. Data from the survey were analysed using STATA version 6.0. ${ }^{11}$ The GP's gender, age, whole time equivalent status, whether or not they held a family planning qualification, and recent attendance at a refresher course were hypothesised to be predictors of GPs' knowledge of EC and prescribing behaviour. The relationship between these five categorical variables was assessed using the chi-squared test for proportions. For each of two outcomes, willingness to prescribe the intra-uterine 
device (IUD) as EC and knowledge of the time limits for insertion of the IUD as EC, odds ratios for the association with each of the five predictor variables were estimated using univariable logistic regression models. To adjust for any potential confounding, a multivariable model with all five explanatory variables was estimated for each outcome. Since responses from GPs within the same practice cannot be considered independent, the chi-squared analyses were adjusted for clustering and all models were estimated in STATA using robust survey estimators.

The free text answers to a question on confidentiality and the under 16's were coded into categories by AG, and independently by DS. Where disagreements existed these were discussed, and a coding category agreed.

\section{Results}

Of the 580 GP principals sent the postal questionnaire, 486 (84\%) responded. Older GPs (over 50 years) were significantly less likely to respond. There were no differences in gender or whole time equivalent status between responders and non-responders (Table 1). There are a total of 145 practices in Avon. No reply was received from six practices - one with two partners and five (out of a total of 16) single-handed practices.

Almost all GPs who responded to the questionnaire provided hormonal EC, only three $(0.6 \%)$ did not. Nearly half of the GPs provided the IUD as EC $(232,47.7 \%)$. Of those who would provide this service, $70(30 \%)$ would do so according to current recommendations, which includes 5 days after expected ovulation as well as up to 5 days after unprotected sex. Almost two thirds of GPs would fit the IUD up to 5 days after unprotected sex $(149,64 \%)$. Eight (4\%) respondents gave an alternative number of days as their answer, and five (2\%) did not complete this section.

Table 1 Characteristics of the sample

\begin{tabular}{llll}
\hline & & $\begin{array}{l}\text { Responders } \\
\text { n } \%)\end{array}$ & $\begin{array}{l}\text { Non } \\
\text { responders } \\
\text { n (\%) }\end{array}$ \\
\hline Sex & Male & $294(60)$ & $64(68)$ \\
& Female & $192(40)$ & $30(32)$ \\
& Total & $486(100)$ & $94(100)$ \\
Age & 30-39 years old & $145(30)$ & $18(19)$ \\
& $40-49$ years old & $215(44)$ & $37(39)$ \\
& $>50$ years old & $126(26)$ & $39(42)$ \\
& Total & $486(100)$ & $94(100)$ \\
Whole time equivalent & & \\
status & & & \\
& Full time & $345(71)$ & $73(77)$ \\
& Three quarter time & $71(15)$ & $12(13)$ \\
& Half time & $70(14)$ & $9(10)$ \\
& Total & $486(100)$ & $94(100)$ \\
\hline
\end{tabular}

In this sample, $399(82 \%)$ GPs held a family planning qualification. Female GPs were significantly more likely to do so compared to male GPs (178, 93\% Vs 221, 76\% $\mathrm{p}<0.001)$. Just under half $(235,48 \%)$ GPs had attended a family planning refresher in the last 5 years. Again, female GPs were more likely to have done so than male GPs $(125 / 186,67 \%$ Vs $110 / 285,39 \%$ p < 0.001). There was also a strong and statistically significant relationship $(\mathrm{p}<0.0001)$ between whole time equivalent status and the GP's gender.

As shown in Table 2, female GPs were significantly more likely than male GPs to fit the IUD as EC, as were GPs with a family planning qualification, and those who had attended
Table 2 Crude and adjusted odds ratios for GPs willing to fit an IUD as $E C$ and whether they do so within the recommended time limits

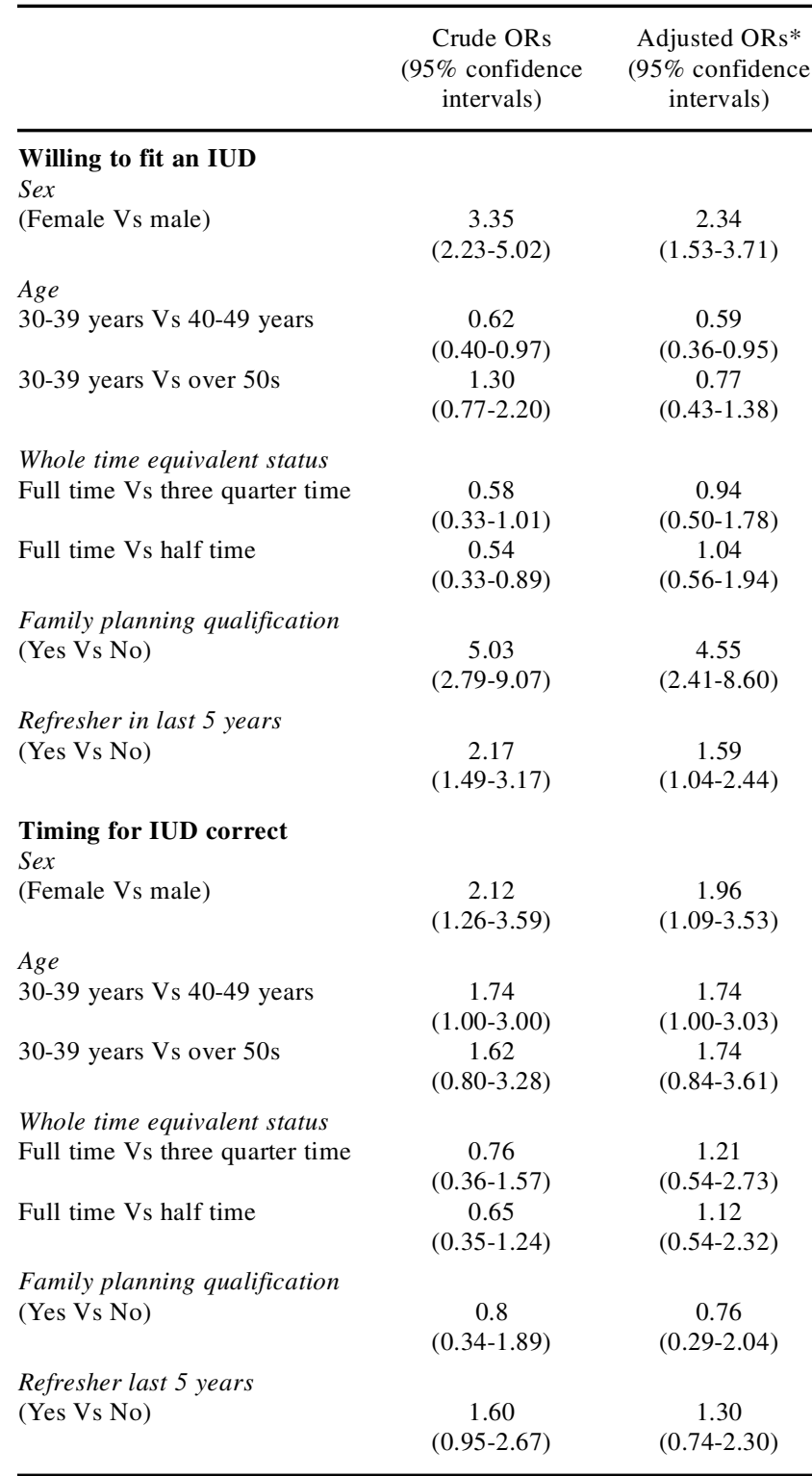

*adjusted for other variables in table and allowing for clustering

a refresher in the last 5 years. GPs were more likely to be willing to fit an IUD as EC if they were in their 40s compared to those in their 30 s or those over 50 . There was no relationship between GPs of differing whole time equivalent status and willingness to provide the IUD as EC in the multivariable model.

GPs were more likely to fit the IUD within the current recommendations for time limits if they were female compared to male GPs. There was some suggestion that GPs in their 40s were more likely than other aged GPs to fit the IUD as EC according to recommended time limits. However, knowledge of the correct time limits for IUD fitting was not affected by the whole time equivalent status of the GP, whether they held a family planning qualification, or had recently attended a refresher course. There was some evidence of confounding in the multivariable analyses when compared to the univariable analyses.

Most GPs $(424,87 \%)$ were willing to provide EC to women not on their practice list. This dependent variable was examined in a multivariable model with the same predictor variables as above, none of the associations were significant. 
GPs were asked: 'If a 14-year-old girl requested EC from you whom, if anyone, would you routinely inform?' The answers were coded into seven categories. The results are shown in Table 3. Eight GPs (1.6\%) would need to obtain consent from an adult.

GPs were also asked if they would provide a 14-year-old with EC on a Sunday, in the event that she had had unprotected sex on the night before and rang for help on the Sunday morning. Half of the sample would see her the same day $(239,49 \%)$. A further quarter of GPs $(113,23 \%)$ would manage by conducting the consultation on the telephone and arranging for her to obtain EC on the Sunday. However, $93(19 \%)$ GPs would ask her to contact the surgery on Monday morning to arrange an appointment to be seen.

Table 3 Coding categories from responses to question: 'If a 14-year-old requested EC from you whom, if anyone, would you routinely inform?'

\begin{tabular}{lll}
\hline Category & $\mathrm{n}$ & $(\%)$ \\
\hline $\begin{array}{l}\text { No one/ No one if 'Fraser ruling competent'/ } \\
\text { Not routinely }\end{array}$ & 248 & $(51)$ \\
Dr would inform parent with patient permission/ & & \\
Dr asks patient to inform parent & 126 & $(26)$ \\
Dr would inform another Dr / Health visitor & 26 & $(5)$ \\
It depends & 33 & $(7)$ \\
Dr would inform a parent & 8 & $(1.6)$ \\
Not applicable & 4 & $(0.8)$ \\
Missing data & 41 & $(8)$ \\
Total & 486 & $(100)$ \\
\hline
\end{tabular}

Access to a family planning trained practice nurse and whether any nurse was involved in providing EC was explored. Twenty-six (5\%) GPs had a nurse available to provide advice and tablets, $92(19 \%)$ to give advice and a script for hormonal EC, 194 (40\%) for advice only, and 156 (32\%) were not involved in EC provision at all. Three quarters $(362,75 \%)$ of GPs had access to a family planning trained practice nurse. A minority of respondents reported that their practice nurse provided advice and a script $(4,0.8 \%)$, or advice only $(26,5.3 \%)$, when their nurse did not have a family planning qualification. However, $74(21 \%)$ of the family planning trained practice nurses were not implicated in the provision of EC.

\section{Discussion}

The sample was chosen in order to provide locally relevant information as part of the development of a health promotion intervention in schools in Avon. There is no reason to believe that Avon is not typical of many other health authorities in the UK.

The survey has limitations as a research methodology. However, we were able to obtain a good response rate, possibly because teenage pregnancy and EC had considerable media coverage during the study period. Older GPs and those from single-handed practices were less likely to respond to this survey, a finding similar to other general practice surveys. ${ }^{12}$ We excluded GP non-principals such as locums, retainers and assistants in general practice, as there were no reliable registers of these doctors available. By excluding this group of doctors, who are mainly female, bias may have been introduced. It is possible that had nonprincipals been included the provision of EC would have been even better than that found.

A study conducted in 1993 by Walsh ${ }^{9}$ explored GPs' views on EC. This study randomly selected 200 GPs from the UK and obtained a $63 \%$ response rate. Our study, and that of Walsh, provide similar results for the provision of both hormonal and IUD EC. However, Walsh found fewer practice nurses involved in the provision of EC (52\%). She also found that less than half $(46 \%)$ of the responding GPs were willing to provide EC to a woman not on their own practice list, and less than three quarters (72\%) would provide EC to a woman under 16 without parental consent.

A minority of GPs in Avon has never received a family planning qualification. GPs of both sexes provide contraceptive advice. Those in their 40s, those with a family planning qualification and those who have attended a refresher in the last 5 years, are more likely to provide and fit the IUD as EC. However, under half have attended a refresher in the last 5 years. It appears that female GPs are more likely to provide the IUD as EC, and to do so within the limits set by the Faculty of Family Planning. ${ }^{13}$ Women GPs are also more likely to have a family planning qualification and to have attended a refresher in the last 5 years compared with male GPs. Female GPs probably see more patients requiring contraceptive advice than their male colleagues, which may well influence these factors. GPs in their 40s are more willing to fit the IUD as EC. This age group may be more likely to have been trained to fit IUDs in an era when family planning clinics were more prevalent compared to older and younger cohorts of GPs.

In our survey only eight GPs, from a sample of 486, were not willing to provide hormonal EC without parental consent. In future research it may be useful to explore GPs' views on this issue in greater depth. However, it does appear that the vast majority of GPs are following the guidance from Lord Fraser produced following the House of Lords ruling in the case of Victoria Gillick Vs West Norfolk and Wisbech Area Health Authority ${ }^{14}$ in the mid 1980s (Figure 1). Guidance on confidentiality and people under 16 was issued to all GPs in $1994 .{ }^{15}$ At the time, a call was made to instigate a communications strategy so that young people know that they can put their trust in doctors in general practice. ${ }^{16}$ This may explain the larger proportion of GPs willing to provide confidential contraceptive advice to the under 16's compared to those surveyed by Walsh ${ }^{9}$ in 1993. We would encourage GPs to promote their confidential contraceptive advice services widely, as recommended by the Social Exclusion Unit's Teenage Pregnancy Report. ${ }^{2}$

There is recent evidence that hormonal EC is more effective if given within 24 hours of unprotected sexual intercourse. ${ }^{6}$ This may explain the intended action of over two thirds of the GPs to provide EC on a Sunday to a woman who has had unprotected sexual intercourse on the Saturday night, rather than waiting until Monday morning. The latter could be a school day, and requires the young person to make another contact with health services; some may lack the motivation to do so.

Figure 1 Criteria for a doctor to provide contraceptive advice or treatment to young people under $16^{*}$

1. The young person understands the doctor's advice.

2. The doctor cannot persuade the young person to inform his or her parents or allow the doctor to inform the parents that he or she is seeking contraceptive advice.

3. The young person is very likely to begin or continue having intercourse with or without contraceptive treatment.

4. Unless he or she receives contraceptive advice or treatment, the young person's physical or mental health or both are likely to suffer.

5. The young person's best interests require the doctor to give contraceptive advice, treatment or both without parental consent.

* Guidelines from Lord Fraser ${ }^{14}$ 
Family planning trained practice nurses are employed by three-quarters of the GPs in this survey. This is a significant increase compared to that found in a survey of Avon GPs undertaken in $1989,{ }^{17}$ when $47 \%$ of GPs had access to a specialist nurse. Practice nurses have been noted to be an under used resource in the area of EC provision in the past. ${ }^{9,10}$ There is a suggestion in this study that although this is still the case, more practice nurses are involved in the provision of EC. However, a fifth of trained family planning practice nurses are not involved at all, and a few practice nurses with no specialist qualifications are. Primary Care Groups may wish to explore this issue and set recommendations locally.

\section{Conclusions}

We believe this survey demonstrates that GPs in Avon are providing a reasonably good service in the provision of EC, with the possible exception of under use of skilled nursing staff. Previous research suggests that a consultation for EC in general practice is used as an opportunity to discuss future contraceptive needs. ${ }^{10}$ This survey was conducted prior to deregulation of hormonal EC. This move was unpopular with both GPs ${ }^{10}$ and pharmacists. ${ }^{18}$ Our results provide evidence that young people, should they choose to obtain hormonal EC free from their own, or another GP, will be provided with a good service.

\section{Acknowledgements}

The authors would like to thank all GPs who took the time to complete the questionnaire. We would also like to acknowledge the support of other members of the Steering Committee for the study; Professor Ian Diamond, Dr Nona Dawson, Mr David Pearson and Dr Hilary Cooling.

Statements on funding and competing interests

Funding. Dr Graham is funded by a South West NHS R\&D Studentship. Additional funds were obtained from the Royal College of General Practitioners' Scientific Foundation Board

Competing interests. None.

References

Conceptions to women under 18 in England and Wales, 1995-97: local authority areas Population Trends 97 Autumn 1999.

Wellings K Fiold J, Joenage Pregnancy. London: HMSO, 1999. 1993; 307: 25 .

Vasilakis C, Jick S, Jick H. The risk of venous thromboembolism in users of postcoital contraceptive pills. Contraception 1999; 59: 79-83.

6 Task Force on Postovulatory Methods of Fertility Regulation. Randomised controlled trial of levonorgestrel versus the Yuzpe regimen of combined oral contraceptives for emergency ontraception. Lancet 1998; 352: 428-433.

Trussell J, Koenig J, Ellertson C, et al. Preventing unintended pregnancy: The costeffectiveness of three methods of emergency contraception. American Journal of Public

Egg Research and Consultancy "You think they won't tell anyone, well you HOPE they won't..." Do young people believe sex advice is confidential? Report commissioned by Broo

postcon coneral practitioners and hospital accid

10 Ziebland S, Graham A, McPherson A. Concerns and cautions about prescribing and deregulating emergency contraception: a qualitative study of GPs using telephone interviews. Family Practice 1998; 15: 449-456.

StataCorp. Stata Statistical Software: Release 6.0. College Station, TX: Stata Corporation. 1999.

12 Templeton L, Deehan A, Taylor C, et al. Surveying general practitioners: does a low response rate matter? British Journal of General Practice 1997; 47: 91-94.

Faculty of Family Planning and Reproductive Health Care of the Royal College of Obstetricians and Gynaecologists. Recommendations for clinical practice: emergenc contraception. London. Royal College of Obstetricians and Gynaecologists, April 2000

Confidentiality and people under 16. Guidance issued jointly by the BMA, GMSC, HEA, Brook Confidentiality and people under 16. Guidd

Scally G Confidentiality, contraception, and young people. British Medical Journal 1993 307: $1157-1158$.

Hine C. Survey of Avon general practitioner family planning services. Bristol and Weston Health Authority Working Party on Family Planning Services: 1989

18 Blackwell D, Taylor G, Holden K. Pharmacists' concerns and perceived benefits from the deregulation of hormonal emergency contraception (HEC). British Journal of Family Planning 1999; 25: 100-104. 